\section{Spontaneous orgasms - an epileptic case without structural correlate}

Over 10 years ago, Verghese (1989) reported on spontaneous orgasms, stating that in the absence of gynaecological, hormonal, or overt psychological disturbances such symptoms might be caused by epileptic seizures as part of an organic brain disease (Ruff, 1980, Remillard et al, 1983, Reading \& Will, 1997). Only a few individual reports have been published on this condition, giving accounts of structural lesions in the temporal or parietal lobe. Erotic manifestations in these papers so far were described as unpleasant sensations.

We describe the case of a 37-year-old female patient who experienced her first generalised tonic-clonic seizure at the age of 28. Not until five years later, when she had her second generalised seizure, did other 'funny' symptoms appear, which she did not recognise as epileptic manifestations. She described epigastric feelings, sensations of déjà $v u$, déjà vécu and depersonalisation as well as spontaneous orgasms, the latter of which was experienced as a pleasant, although embarrassing, sensation. These feelings could occur anywhere, at home, at work or on entering a bus. During those events she often was able to continue in her work or in her conversations, paleness being the only noticeable symptom. The frequency of such episodes ranged between one to six per month. Full diagnostic work-up was only performed three years later when she had had her third generalised seizure. As on the previous occasions her general, neurological and psychiatric examinations were normal. Computed tomography scan, magnetic resonance and electroencephalogram (EEG) were found free of structural lesions and focal signs, but a long-term EEG revealed a right temporal sharp-wave focus lasting five seconds, indicating that the symptoms of our patient could be identified as part of an epileptic aura pointing towards partial seizures with an origin in the right temporal lobe. This diagnosis was confirmed by the fact that all symptoms ceased after initiating antiepileptic treatment with lamotrigine.

This case suggests that neuroimaging might not always be sensitive enough to reveal structural lesions, and that long-term EEG recordings should be included in the diagnostic work-up. In doubtful cases a trial with antiepileptics can prove helpful. A thorough history-taking should always include questions about other concomitant epileptic symptoms. Whether erotic manifestations during partial epileptic seizures are experienced as being pleasant or unpleasant, they still are embarrassing and a burden for the patient and therefore warrant pharmacological treatment.

Reading, P. J. \& Will, R. G. (1997) Unwelcome orgasms. Lancet, 350, 1746.

Remillard, G. M., Andermann, F., Testa, G. F., et al (1983) Sexual ictal manifestations predominate in women with temporal lobe epilepsy: A finding suggesting sexual dimorphism in the human brain. Neurology, 33, 323-330.

Ruff, R. L. (1980) Orgasmic epilepsy. Neurology, 30 1252-1253.

Verghese, C. (1989) Spontaneous orgasms - an explanation? British Journal of Psychiatry, I55, 870.

R. Crevenna Department of Physical Medicine, University of Vienna Medical School,Währinger Gürtel I8-20, A-I090 Vienna, Austria

N. Homann, M. Feichtinger, E. Ott, E. Körner Department of Neurology, Karl Franzens University Medical School, Graz, Austria

\title{
One hundred years ago
}

\section{The Metropolitan Asylums Board and its Assistant Medical Officers. To the Editors of The Lancet}

Sirs, In The Lancet of Jan. 13th there appeared an advertisement for an assistant medical officer at the Caterham Asylum of the Metropolitan Asylums Board. Having had experience of that Board I should like to point out for the benefit of intending applicants a few of the conditions under which the assistant medical officers at their asylums work. On entering upon my duties I was taken to my quarters by the steward, who apologised for the lack of accommodation offered me, explaining at the same time that such conditions were only temporary. My "quarters" consisted of one room in which I slept, the same room had also to serve me as an office, dining-room, as well as for the reception of personal visitors, and indeed for every use that it is possible to put a room to. All that was temporary as far as I was concerned consisted in the facts that on committee days a screen was taken from the room, so that visitors could see the bed and the fact that I had a knob put on the door after I had occupied my quarters for three weeks. Since I left there have been improvements at that particular asylum. The junior assistant medical officer now shares in a common dining-room and has consequently only to use his bedroom for an office and living-room generally. The medical superintendents at their asylums are not allowed to write testimonials. The Metropolitan Asylums Board, however, accept testimonials from other asylums. Such conditions prevailing one is not surprised to find that after years of service under the Board promotion is impossible elsewhere, and as the Board is not enamoured with the idea of promoting its own men the assistant medical officers find themselves in an awkward position. At other asylums it is the practice to allow the assistant medical officer the use of spare bedrooms in which to lodge a visitor, it being recognised that life in asylums is somewhat monotonous.
In my time when a visitor arrived his host laid himself down on his couch (if he had one, I had not) and with his clothes on was happy that his brother from Scotland or a medical friend from America had come to see him. They were the only visitors whilst I served the Metropolitan Asylums Board. Now, the Metropolitan Asylums Board will no longer allow any visitor to be the guest of their assistant medical officer overnight.

Would intending applicants for the post advertised kindly apply to any of the Metropolitan Asylums Board's medical officers for information?

I am, Sirs, yours faithfully,

F.J.S.

\section{REFERENCE}

Lancet, 20 January 1900, 211.

Researched by Henry Rollin, Emeritus Consultant Psychiatrist, Horton Hospital, Epsom, Surrey 